\title{
Paramagnetic Resonance as a Probe of the Spin Gap in Normal State of $\mathrm{Hg}_{.77} \mathrm{~Pb}_{.33} \mathrm{Ba}_{2} \mathrm{Ca}_{2} \mathrm{Cu}_{3} \mathrm{O}_{8+x}$ Superconductor
}

\author{
Frank J. Owens \\ Department of Physics, Hunter College, CUNY, 695 Park Avenue, New York, NY 10065, USA \\ Correspondence should be addressed to Frank J. Owens, owensfj@gmail.com
}

Received 30 August 2011; Accepted 26 September 2011

Academic Editors: S. Balamurugan and J. Bok

Copyright ( $\odot 2011$ Frank J. Owens. This is an open access article distributed under the Creative Commons Attribution License, which permits unrestricted use, distribution, and reproduction in any medium, provided the original work is properly cited.

\begin{abstract}
It is shown that electron paramagnetic resonance (EPR) can be used to observe the spin gap in copper oxide superconductors. The electron paramagnetic resonance spectra of the $\mathrm{Cu}^{2+}$ ion in underdoped $\mathrm{Hg}_{.77} \mathrm{~Pb}_{33} \mathrm{Ba}_{2} \mathrm{Ca}_{2} \mathrm{Cu}_{3} \mathrm{O}_{8+x}$ show a pronounced decrease in intensity in the normal state as the temperature is lowered to $133 \mathrm{~K}$, the superconducting transition temperature of the material. The decrease is attributed to a pairing of the $\mathrm{Cu}^{2+} s=1 / 2$ spins to form a spin gap. A spin gap of $0.0533 \mathrm{eV}$ is estimated from the data which is in order of magnitude agreement with values obtained from NMR measurements.
\end{abstract}

\section{Introduction}

It is well established that a spin gap, that is, a noncoherent pairing of the $s=1 / 2$ copper spins to form a singlet, exists above $T_{c}$ in the underdoped $\mathrm{YBa}_{2} \mathrm{Cu}_{3} \mathrm{O}_{6.7}$ superconductor. The spin gap is manifested as a change in the slope of the temperature dependence of the resistance and susceptibility above $T_{c}[1,2]$. More definitive evidence comes from angleresolved photoemission spectroscopy which shows a shift of the spectral intensity away from the Fermi level above $T_{c}$ in the underdoped material [3].

The question of the existence of the spin gap in other underdoped copper oxide superconductors has received less attention. The $N=3$ phase of the $\mathrm{HgBa}_{N-1} \mathrm{Ca}_{N-1} \mathrm{Cu}_{N} \mathrm{O}_{2 N+2+x}$ superconductor having a $T_{c}$ of $133 \mathrm{~K}$ is a candidate for the existence of a spin gap above $T_{c}$. Measurements of the pressure dependence show an increase in $T_{c}$ with pressure which is generally accepted to imply that the phase is underdoped [4]. Here the existence of a spin gap above $T_{c}$ in $\mathrm{Hg}_{.77} \mathrm{~Pb}_{.33} \mathrm{Ba}_{2} \mathrm{Ca}_{2} \mathrm{Cu}_{3} \mathrm{O}_{8+x}(\mathrm{Hg}, \mathrm{Pb} 1223)$ is suggested by a change in slope of the temperature dependence of the resistivity and surface resistance above $T_{c}$. However, the main focus of this work is to show that the temperature dependence of the electron paramagnetic resonance (EPR) of the $\mathrm{Cu}^{2+}$ ion in $\mathrm{Hg}_{.77} \mathrm{~Pb}_{.33} \mathrm{Ba}_{2} \mathrm{Ca}_{2} \mathrm{Cu}_{3} \mathrm{O}_{8+x}$ provides evidence for the existence of a spin gap in the material and allows an estimate of its magnitude. It has previously been shown in $\mathrm{Co}$-doped $\mathrm{YBa}_{2} \mathrm{Cu}_{3} \mathrm{O}_{6.7}$ that electron paramagnetic resonance can detect the spin gap, because of the formation of $\mathrm{Co}-\mathrm{Cu}$ singlet state causing a reduction in the intensity of the Co EPR signal strength with decreasing temperature in the normal state [5]. However, a more accurate measurement of the spin gap would be obtained from measuring the decrease in the $\mathrm{Cu}^{2+}$ resonance. In $\mathrm{YBa}_{2} \mathrm{Cu}_{3} \mathrm{O}_{7-x}$, the EPR signal of $\mathrm{Cu}^{2+}$ is not observed in the optimally doped material but is detected in the underdoped material [6-8]. Thus we should expect in $\mathrm{Hg}_{.77} \mathrm{~Pb}_{33} \mathrm{Ba}_{2} \mathrm{Ca}_{2} \mathrm{Cu}_{3} \mathrm{O}_{8+x}$, which has a $T_{c}$ of $133 \mathrm{~K}$ and is underdoped, an EPR of the $\mathrm{Cu}^{2+}$ intrinsic to the superconducting phase.

\section{Experimental}

An E-9 Varian electron paramagnetic resonance spectrometer operating at $9.2 \mathrm{GHz}$ was used to study the derivative of the resonance absorption as a function of dc magnetic field. The samples were contained in and cooled using a doublewalled quartz finger Dewar which is inserted through holes on the top and bottom of the cavity and through which cold $\mathrm{N}_{2}$ or He gas flows. This system enables control of the temperature, monitored by a gold-chromel thermocouple in contact with the sample, to within $\pm 1 \mathrm{~K}$. Surface resistance measurements at $9.2 \mathrm{GHz}$ were made using a microwave 


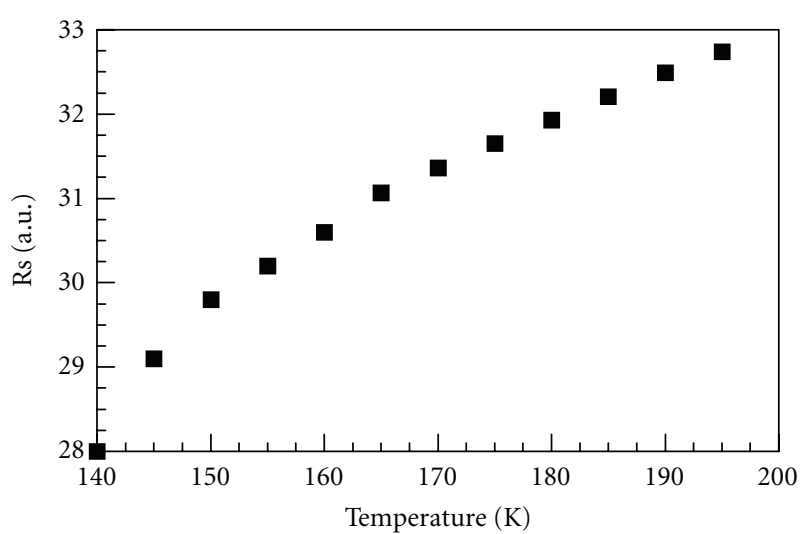

Figure 1: A measurement of the temperature dependence of the surface resistance in the normal state showing a deviation from linearity at $170 \mathrm{~K}$ suggestive of the possibility of the existence of a spin gap.

bridge system previously described which is essentially the bridge of the electron spin resonance spectrometer with the modulation turned off [9]. The samples were synthesized by a two-step process previously described [10]. X-ray diffraction of the samples indicated that the material was $100 \% N=3$ phase, and temperature-dependent resistance and susceptibility data indicated a $T_{c}$ of $133 \mathrm{~K}[10]$.

\section{Results and Discussion}

An examination of previously published temperature-dependent resistance data in the normal state shows an increased negative slope and a deviation from linearity in the vicinity of $180 \mathrm{~K}$ which has been argued to be evidence for the existence of a spin gap in the material. The effect can also be seen in the temperature dependence of the surface resistance in the normal state shown in Figure 1. There is a clear deviation from linearity at $170 \mathrm{~K}$ well above $T_{c}$ which suggests the existence of a spin gap.

Figure 2 shows the EPR spectrum in pellets of $\mathrm{Hg}$, $\mathrm{Pb} 1223$ at room temperature and below $T_{c}$ at $118 \mathrm{~K}$. Two powder spectra are observed, a sharp spectrum having an axially symmetric $g$ tensor and at lower magnetic field a larger broad line having a g value of 2.432 at room temperature which is appropriate to $\mathrm{Cu}^{2+}$. This latter spectrum is attributed to the $\mathrm{Cu}^{2+}$ ions in the superconducting grains. The large width of the line is likely a result of dipolar broadening due to magnetic interactions between neighboring $\mathrm{Cu}^{2+}$ ions. The narrow spectrum is attributed to an impurity in the material and is not associated with the superconducting phase. The marked decrease in the intensity of the broad line in the superconducting phase, in contrast to the increase in intensity of the narrow line, is evidence that the broad line arises from superconducting grains. This occurs because of the reduction of the penetration depth of the dc magnetic field into the sample in the superconducting state. In effect, the dc magnetic field only probes paramagnetic entities in the surface of the sample within the penetration depth. Note also there is a significant shift of the resonance to lower magnetic

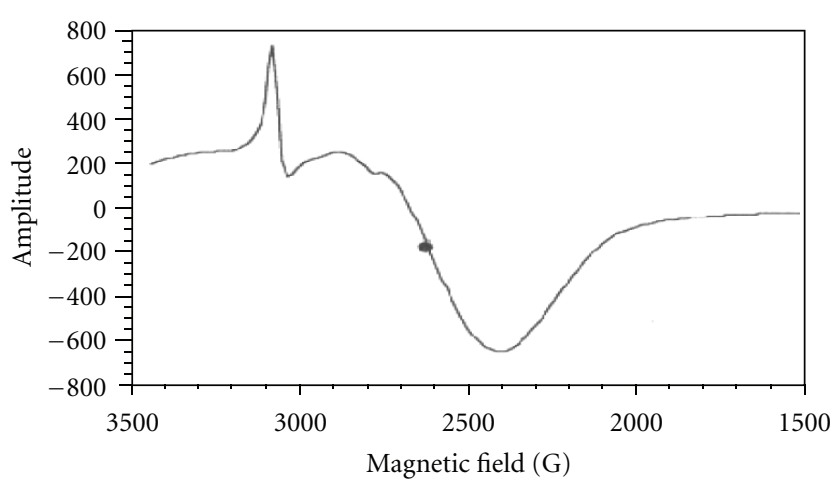

(a)

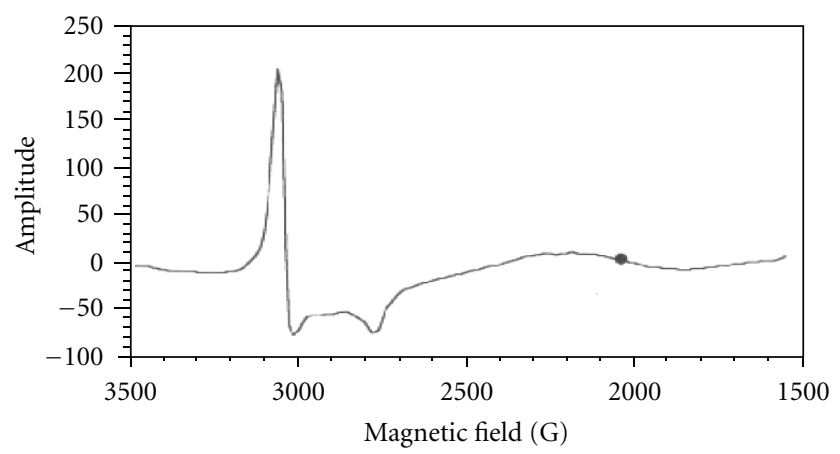

(b)

Figure 2: EPR spectra at room temperature (a) in $\mathrm{Hg}_{.77} \mathrm{~Pb}_{33} \mathrm{Ba}_{2} \mathrm{Ca}_{2} \mathrm{Cu}_{3} \mathrm{O}_{8+x}$ and at $118 \mathrm{~K}$, (b) in the superconducting state showing the markedly reduced intensity of the lower field resonance.

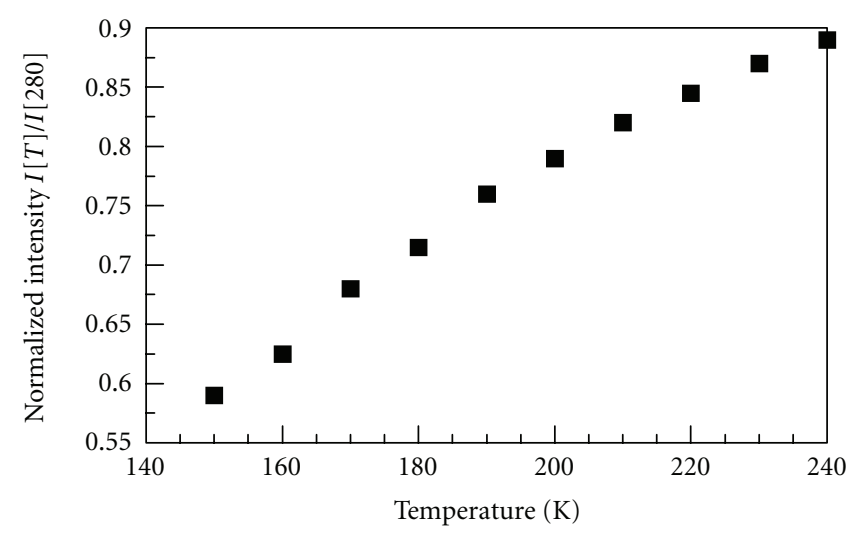

Figure 3: Plot of the temperature dependence of the intensity of the broad line normalized to the Boltzman-temperature-dependent increase that occurs when the temperature is lowered.

field value (increased $g$ value). This shift occurs gradually as a function of temperature in the normal state. Such shifts have been observed in low-dimensional antiferromagnetic materials and shown to be due to antiferromagnetic spin ordering fluctuations $[11,12]$. Figure 3 shows a measurement of the intensity of the broad line, corrected for the intensity increase due to the Boltzman population factor with decreasing temperature in the normal state. This decrease in 


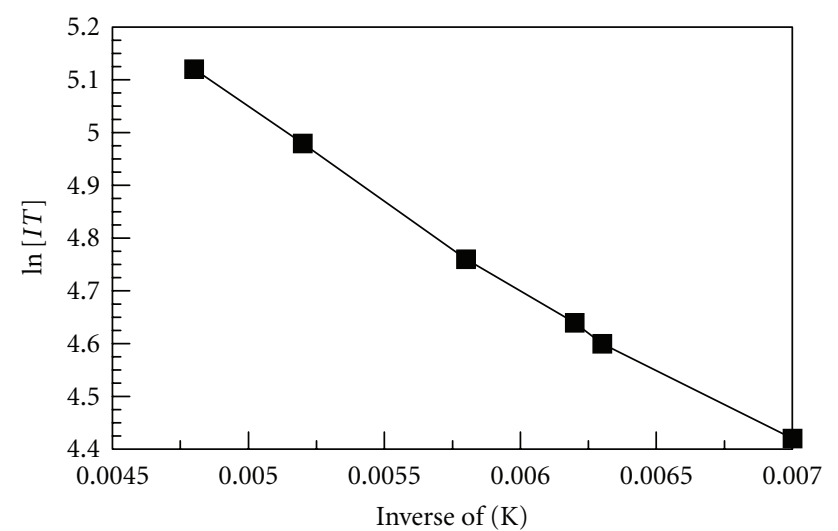

Figure 4: Plot of $\ln [I T]$ versus $1 / T$ where $I$ is the normalized intensity of the EPR signal.

the intensity of the EPR signal with lowering temperature cannot be accounted for by the decrease in the surface resistance which is much smaller per degree than the decrease in the EPR signal strength. This decrease in intensity with lowering temperature in the normal state is attributed to the formation of nonparamagnetic singlet pairs between adjacent $\mathrm{Cu}^{2+}$ ions in effect a spin gap. For a one-dimensional chain or ladder, the intensity $I$ of the EPR signal has been shown to depend on temperature in the presence of a spin gap, $\Delta$, as $[13,14]$

$$
I=\frac{[C \exp (-\Delta / T)]}{T^{N}}
$$

where $N=1$ for a chain and $N=1 / 2$ for a ladder. No analytical expression has been developed for a two-dimensional array. It has been shown that the cuprate superconductors contain stripes which are one-dimensional rows of antiferromagnetic order of $\mathrm{Cu}^{2+}$ spins separated by rows of holes [15]. Thus, in effect, there is a one-dimensional antiferromagnetic ordering of $\mathrm{Cu}^{2+}$ spin so that equation (1) having $N=1$ may be applicable. Figure 4 is a plot of the $\ln (I T)$ versus $1 / T$ which is fit to a straight line allowing an estimate of the spin gap which is obtained to be $0.0533 \mathrm{eV}$ in order of magnitude agreement with the NMR measurement [16]. The EPR measurement of the spin gap is somewhat smaller than the NMR measurement which may be due to the fact that the EPR measurement was on Pb-doped material, whereas the NMR measurement was not. The fact that a straight line results suggests that the $\mathrm{Cu}^{2+}$ pairing is one dimensional consistent with the existence of stripes.

In conclusion, an EPR spectrum of $\mathrm{Cu}^{2+}$ from the superconducting grains of $\mathrm{Hg}, \mathrm{Pb} 1223$ shows a marked decrease in intensity in the normal state as the temperature is lowered to $T_{c}$ which is attributed to the opening of a spin gap in the underdoped material. The spin gap is estimated from the data.

\section{References}

[1] T. Ito, K. Takenaka, and S. Uchida, "Systematic deviation from $\mathrm{T}$-linear behavior in the in-plane resistivity of $\mathrm{YBa}_{2} \mathrm{Cu}_{3} \mathrm{O}_{7-\mathrm{y}}$ : evidence for dominant spin scattering," Physical Review Letters, vol. 70, no. 25, pp. 3995-3998, 1993.

[2] B. Bucher, P. Steiner, J. Karpinski, E. Kaldis, and P. Wachter, "Influence of the spin gap on the normal state transport in $\mathrm{YBa}_{2} \mathrm{Cu}_{4} \mathrm{O}_{8}$," Physical Review Letters, vol. 70, no. 13, pp. 20122015, 1993.

[3] A. G. Loser, Z. X. Shen, and D. S. Dessau, "Doping dependence of $\mathrm{Bi}_{2} \mathrm{Sr}_{2} \mathrm{CaCu}_{2} \mathrm{O}_{8+\sigma}$ in the normal state," Physica C, vol. 263, no. 1-4, pp. 208-213, 1996.

[4] M. Nuñez-Regueiro, J. L. Tholence, E. V. Antipov, J. J. Capponi, and M. Marezio, "Pressure-induced enhancement of $\mathrm{T}_{c}$ above $150 \mathrm{~K}$ in Hg-1223," Science, vol. 262, no. 5130, pp. 97-99, 1993.

[5] F. J. Owens, "Paramagnetic resonance evidence for the opening of a spin gap in $\mathrm{YBa}_{2} \mathrm{Cu}_{2.7} \mathrm{Co}_{0.3} \mathrm{O}_{7-x}$," Physica $C$, vol. 325, no. 1-2, pp. 23-26, 1999.

[6] F. J. Owens, B. L. Ramakrishna, and Z. Iqbal, "EPR in $\mathrm{YBa}_{2} \mathrm{Cu}_{3} \mathrm{O}_{7-\delta}$ and $\mathrm{YBa}_{2} \mathrm{Cu}_{3} \mathrm{O}_{6+x}$," Physica $C$, vol. 156 , no. 2, pp. 221-224, 1988.

[7] F. Mehran, S. E. Barnes, T. R. McGuire, T. R. Dinger, D. L. Kaiser, and F. Holtzberg, "Observation by electron spin resonance of a pseudo-cubic site in $\mathrm{YBa}_{2} \mathrm{Cu}_{3} \mathrm{O}_{7-x}$," Solid State Communications, vol. 66, no. 3, pp. 299-302, 1988.

[8] J. Albino, O. de Aguiar, A. A. Menovsky, J. van den Berg, and H. B. Brom, "ESR in $\mathrm{YBa}_{2} \mathrm{Cu}_{3} \mathrm{O}_{7-\delta}$ crystals," Physica C, vol. 21, no. 8, p. L237, 1988.

[9] F. J. Owens, A. G. Rinzler, and Z. Iqbal, "Magneto-microwave absorption study of flux depinning in $\mathrm{Hg}_{0.7} \mathrm{~Pb}_{0.3} \mathrm{Ba}_{2} \mathrm{Ca}_{2} \mathrm{Cu}_{3} \mathrm{O}_{8}$ superconductor," Physica C, vol. 233, no. 1-2, pp. 30-34, 1994.

[10] Z. Iqbal, T. Datta, D. Kirven et al., "Superconductivity above $130 \mathrm{~K}$ in the $\mathrm{Hg}-\mathrm{Pb}-\mathrm{Ba}-\mathrm{Ca}-\mathrm{Cu}-\mathrm{O}$ system," Physical Review $\mathrm{B}$, vol. 49, no. 17, pp. 12322-12325, 1994.

[11] K. Oshima, K. Okuda, and M. Date, "G-shift in low dimensional antiferromagnets at low temperatures," Journal of the Physical Society of Japan, vol. 41, no. 2, pp. 475-480, 1976.

[12] Y. Morimoto and M. Date, "Anomalous shift of ESR lines in two dimensional antiferromagnet $\mathrm{Cu}(\mathrm{HCOO})_{24} \mathrm{H}_{2} \mathrm{O}$," Journal of the Physical Society of Japan, vol. 29, no. 4, p. 1093, 1970.

[13] L. N. Bulaevskii, A. I. Buzdin, and D. I. Khomskii, "Spinpeierls transition in magnetic field," Solid State Communications, vol. 27, no. 1, pp. 5-10, 1978.

[14] M. Troyer, H. Tsunetsugu, and D. Würtz, "Thermodynamics and spin gap of the Heisenberg ladder calculated by the lookahead Lanczos algorithm," Physical Review B, vol. 50, no. 18, pp. 13515-13527, 1994.

[15] J. M. Tranquada, B. J. Sternlieb, J. D. Axe, Y. Nakamura, and S. Uchida, "Evidence for stripe correlations of spins and holes in copper oxide superconductors," Nature, vol. 375, no. 6532, pp. 561-563, 1995.

[16] M. H. Julien, P. Carretta, M. Horvatić et al., "Spin gap in $\mathrm{HgBa}_{2} \mathrm{Ca}_{2} \mathrm{Cu}_{3} \mathrm{O}_{8+\delta}$ single crystals from 63Cu NMR," Physical Review Letters, vol. 76, no. 22, pp. 4238-4241, 1996. 

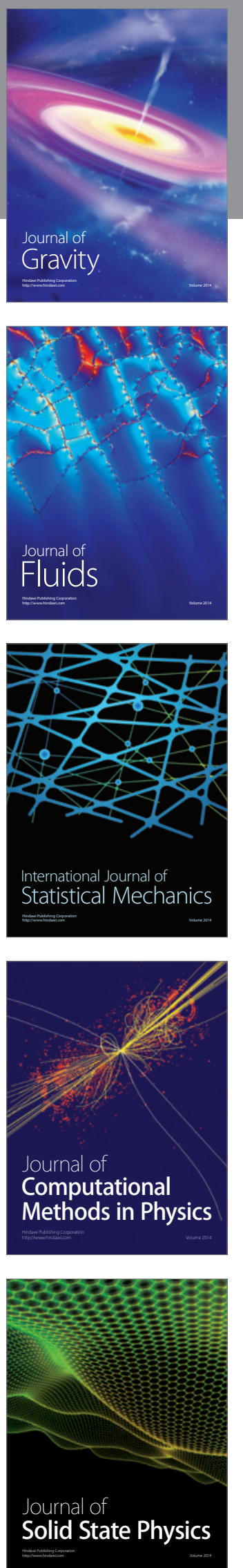
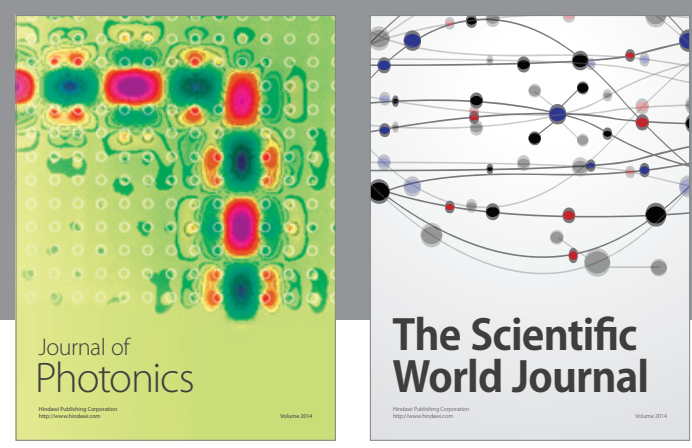

The Scientific World Journal

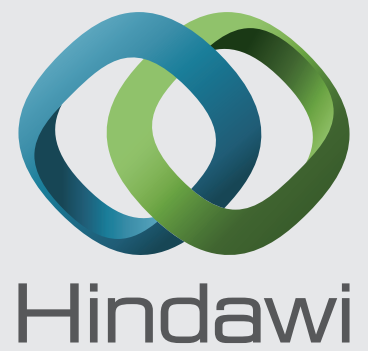

Submit your manuscripts at http://www.hindawi.com
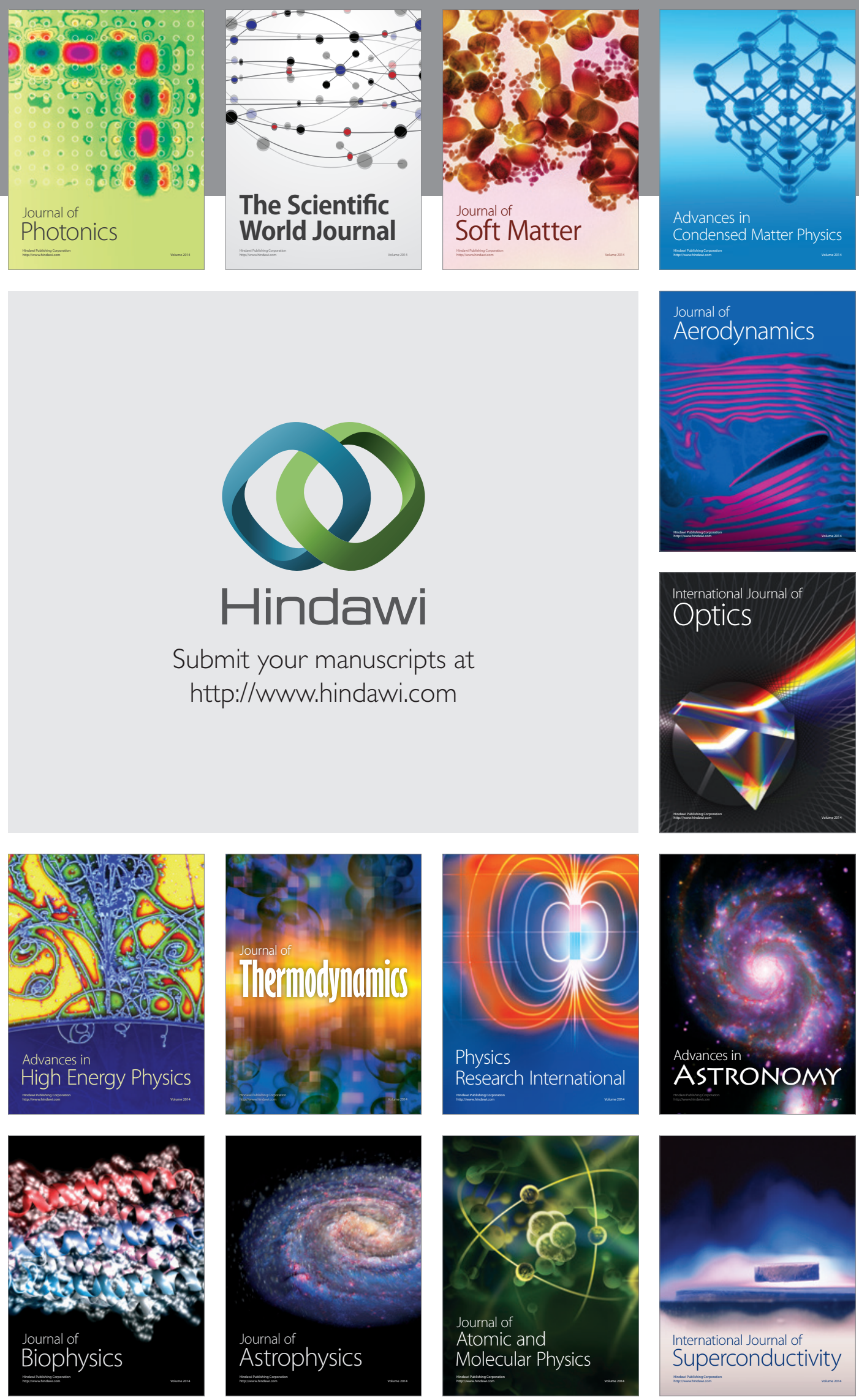
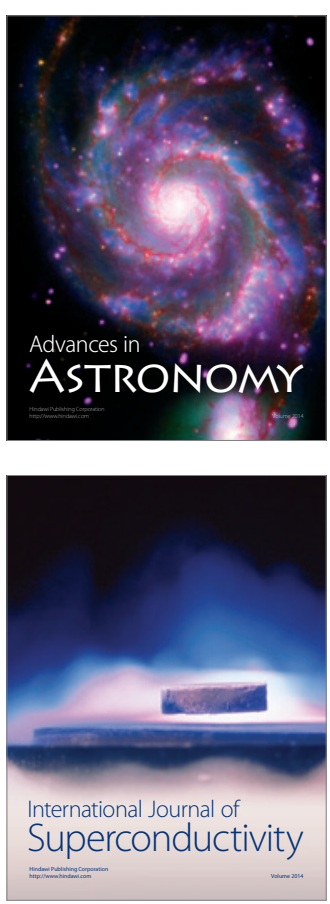